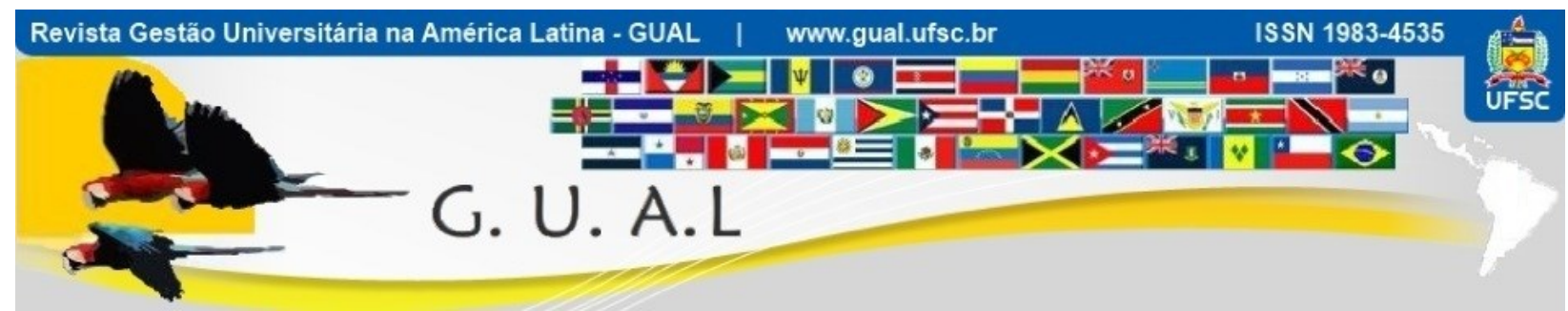

DOI: http://dx.doi.org/10.5007/1983-4535.2018v11n3p235

\title{
O COMPROMETIMENTO E SEUS EFEITOS SOBRE A SATISFAÇÃO DE CLIENTES, A INTENÇÃO EM DEFENDER A MARCA E A INTENÇÃO DE RECOMPRA EM SERVIÇOS DE ENSINO
}

\author{
COMMITMENT AND IT'S EFFECTS ON CUSTOMER SATISFACTION ON \\ ADVOCACY INTENTIONS AND REPURCHASE INTENTION IN EDUCATION \\ SERVICES
}

Vinicius Zanchet de Lima, Doutorando Universidade de Caxias do Sul - UCS vinizanchet@gmail.com

Gabriel Sperandio Milan, Pós-doutor Universidade de Caxias do Sul - UCS gabmilan@terra.com.br

Ana Paula Graciola, Doutoranda Universidade de Caxias do Sul - UCS anagraciola@gmail.com

Deonir De Toni, Pós-doutor Universidade de Caxias do Sul - UCS deonirdt@terra.com.br

Recebido em 12/janeiro/2018

Aprovado em 05/agosto/2018

Sistema de Avaliação: Double Blind Review

Esta obra está sob uma Licença Creative Commons Atribuição-Uso. 


\title{
RESUMO
}

Nas empresas prestadoras de serviços educacionais, o comprometimento do aluno com a marca da universidade é um diferencial perante a concorrência. O objetivo contemplou verificar qual o impacto do comprometimento afetivo, calculativo e normativo sobre a satisfação do cliente, intenção em defender a marca e a intenção de recompra de serviços educacionais da mesma instituição de ensino superior (IES), avaliando a moderação do gênero nas relações entre os consequentes do comprometimento. Os resultados obtidos, por meio da aplicação de uma pesquisa quantitativa descritiva junto a 283 alunos de graduação de uma instituição de ensino superior, demonstraram que dentre os três componentes do comprometimento, o afetivo é o que mais impacta na satisfação do cliente e na intenção do aluno em defender a marca. Além do mais, foi possível observar que a não confirmação das hipóteses do comprometimento calculativo em relação à satisfação do cliente e a intenção em defender a marca, evidenciam a insatisfação e a não defesa da marca, quando os alunos se sentem refém à ela, fato que, provoca a recusa de recomprar novamente um novo serviço. $\mathrm{O}$ efeito moderador do gênero nas relações contempladas mostrou-se não ser significativo.

Palavras chave: Comprometimento. Satisfação do Cliente. Intenção em Defesa a Marca. Intenção de Recompra. Serviços Educacionais.

\begin{abstract}
In service education firms, student commitment with the brand of the university is a differentiator from the competitors. This paper aimed to verify the impact of affective, calculative and normative commitment on customer satisfaction, advocacy intentions and intention repurchase of educational services in the same higher education institution (HEI), evaluating the moderation of gender in the relationship between consequent commitment. The results obtained through the application of a descriptive quantitative survey of 280 undergraduate students of a higher education institution, showed that among the three components of commitment, affection is the one that most impact on customer satisfaction and intention of the student advocacy intentions. Moreover, it was observed that the failure to confirm the hypothesis of calculative commitment, regarding the customer satisfaction and advocacy intention, highlight the dissatisfaction and no advocacy intentions, when customers feel hostage to it, fact that causes the refusal to repurchase the service again. The moderating effect of gender in the relationships considered proved not to be significant.
\end{abstract}

Keywords: Commitment. Customer Satisfaction. Advocacy Intentions. Repurchase Intention. Educational Service. 


\section{INTRODUÇÃO}

Em um ambiente de concorrência, compreender os processos de tomada de decisão dos clientes para predizer as suas intenções e os seus comportamentos futuros tem sido o objetivo de alguns estudos na área de marketing, principalmente associados às teorias do comportamento do consumidor (HAN; HSU; SHEU, 2010; HAN; KIM, 2010). Um dos principais componentes no desenvolvimento e manutenção do ralacionamento ao longo prazo, é o comprometimento (FULLERTON, 2003; MORGAN; HUNT, 1994). Da mesma forma, no fluxo de relacionamento com o cliente, o comprometimento é reconhecido como um precursor essencial nas intenções futuras (BANSAL; IRVING; TAYLOR, 2004) e lucratividade (ANDERSON; WEITZ, 1992). Considerado como um tema central na área de marketing, ou seja, uma das construções mais estudadas em relacionamentos de mercado (MORGAN; HUNT, 1994; PALMATIER et al., 2013).

Os primeiros estudos em marketing abordavam o compromisso (comprometimento) de uma forma unidimensinal (por exemplo, GARBARINO; JOHNSON, 1999; MOORMAN; ZALTMAN; DESHPANDE, 1992) enquanto que estudos posteriores (OZAG, 2006; STANLEY; RHOADES; WHITTON, 2010) conceituaram de uma forma multidimensional, utilizando um modelo com três componentes, ou seja, comprometimento afetivo, comprometimento calculativo e comprometimento normativo. No entanto, o comprometimento multidimensional pode ser o mais adequado para ser mensurado no contexto de serviço (KEININGHAM, et al.,2016). Inclusive, estudos mencionam que a análise do comprometimento, na literatura, em um ambito relacional, apresenta um maior enfoque nos âmbitos afetivos e calculativos, (JONES et al., 2007), e poucos estudos abordaram o comprometimento de uma forma multidimensional utilizando os três componentes, afetivo, normativo e calculativo (BANSAL; IRVING; TAYLOR, 2004).

Portanto, esta pesquisa emprega uma estrutura conceitual abrangente, que consiste nas três dimensões do comprometimento, afetivo calculativo e normativo sugerido por Allen e Meyer (1990). Shukla, Banerjee e Singh (2016) trazem contribuições para o estudo sobre o comprometimento (multidimensional) do cliente com a empresa, ao desenvolverem um modelo teórico abordando os antecedentes e consequentes do comprometimento, aplicado no contexto de marcas de luxos. O presente estudo contribui com a verificação da relação dos consequentes do comprometimento abordado pelos autores Shukla, Banerjee e Singh (2016), relacionados com a intenção de recompra no contexto de ensino. Nesse sentido, define-se o 
problema de pesquisa da seguinte forma: qual o impacto do comprometimento afetivo, calculativo e normativo sobre a satisfação do cliente (aluno), a intenção em defender a marca e a intenção de recompra de serviços educacionais da mesma IES? Ademais, o estudo se propõe a avaliar o possível efeito moderador do gênero nas relações entre os consequentes contemplados do comprometimento, haja visto, que estudos mencionam que é necessário o desenvolvimento de novas pesquisas envolvendo a moderação do gênero entre os construtos (CHO; RUTHERFORD, 2011, MITTAL; KAMAKURA, 2001).

Em termos de contribuição acadêmica, o estudo busca trazer contribuições acerca do comprometimento e os seus consequentes contemplados para esta pesquisa, objetivando uma maior consolidação da teoria e ampliando o escopo do estudo para o contexto educacional. A pesquisa propõe ainda, ampliar seus resultados para os profissionais de marketing, à medida que se observa uma competição acirrada no mercado, entender qual é a intenção comportamental dos alunos, torna-se essencial para sobrevivência no mercado. Além disso, com uma melhor compreensão da diferença de género, as prestadoras de serviços educacionais serão capazes de desenvolver e adaptar seus programas de fidelidade para aumentar a intenção de recompra de seus cliente, baseado nas diferenças de género dentro de sua base de clientes. Para apresentar o estudo, estruturalmente, esse trabalho é iniciado pela seção introdutória, seguida pela fundamentação teórica, método de pesquisa, análise dos resultados obtidos e por fim as considerações finais.

\section{REFERENCIAL TEÓRICO}

\subsection{RELAÇÃO ENTRE COMPROMETIMENTO E SATISFAÇÃO DO CLIENTE}

Originado da psicologia social, o conceito de comprometimento está relacionado à associação do desejo do consumidor em continuar uma relação com a empresa e em resistir às ofertas da concorrência (WU; CHEN; CHUNG, 2009), por mais atraentes que sejam.

A construção do comprometimento (ALLEN; MEYER, 1990) e sua literatura emergem da ciência organizacional, onde é empregada para investigar como os funcionários se comprometem com a empresa. Com o enfoque do comprometimento em nível organizacional, Allen e Meyer (1990) preconizaram um modelo de três dimensões: afetivo, instrumental e normativo. A dimensão enfoque afetivo, segundo Gruen, Summers e Acito (2000), é o grau em que o membro e a organização estão ligados, em base em quão bem ele se sente sobre tal organização. Por outro lado, o enfoque instrumental reflete o 
comprometimento do trabalhador com a organização, enquanto existir a percepção de benefícios. Já, a abordagem normativa pressupõe que o comprometimento deriva de um senso de obrigação moral do trabalhador junto à organização (MEYER et al., 2002).

O comprometimento é cada vez mais utilizado nas pesquisas de marketing, principalmente no campo relacional (MORGAN; HUNT, 1994; PALMATIER et al., 2013). Além disso, o comprometimento está intimamente relacionado com as intenções e o comportamento dos clientes, incluindo a satisfação (SHUKLA; BANERJEE; SINGH, 2016). Embora o construto satisfação do cliente seja consolidado na literatura, ainda há poucos estudos que buscaram as relações entre os construtos comprometimento e satisfação do cliente (SU et al., 2016). Oliver (1981, p.27), conceituou a Satisfação do cliente como "uma avaliação da surpresa inerente à aquisição de um produto e/ou à experiência de consumo", inserindo a dimensão afetiva. Anderson, Fornell e Lehmann (1994) mencionam que a satisfação do cliente é compreendida como um precursor do comprometimento dos clientes, por ela ser o resultado das avaliações do cliente em relação ao produto e/ou serviço e se configura como um aspecto essencial para a sua decisão de manter um relacionamento.

Suportado por alguns estudos (MATHIEU, 1991; SHUKLA; BANERJEE; SINGH. 2016), verifica-se que o comprometimento afetivo apresenta uma relação positiva em relação à satisfação do cliente. Ampliando o debate, estudos demonstraram que o comprometimento normativo pode ser construído a partir do conceito de reciprocidade, como uma força de influência em uma situação de troca (MEYER et al., 2002). Esta relação de troca pode gerar satisfação para o cliente (CIALDINI, 2001), uma vez, que a reciprocidade é vista em muitos relacionamentos, como é o caso de amizades, casamentos e outras parcerias (JONES et al., 2010). Neste sentido, Ziaullah, Feng e Akhter (2014) desenvolveram um estudo no contexto de compras online na China, no qual apontou que é consistente a influência positiva do comprometimento normativo sobre a satisfação do cliente. Por outro lado, Gustafsson, Johnson e Roos (2005) e Shukla, Banerjee e Singh (2016) demonstraram que o compromisso calculista tem um impacto negativo sobre a satisfação do cliente, possivelmente devido aos clientes sentir-se preso em um relacionamento, devido à ausência de alternativas ou os custos altos em manter-se no relacionamento. Assim, postula-se as seguintes hipóteses:

H1: O comprometimento afetivo tem impacto positivo na satisfação do cliente.

H2: O comprometimento calculativo tem impacto negativo na satisfação do cliente.

H3: O comprometimento normativo tem impacto positivo na satisfação do cliente. 


\subsection{RELAÇÃO ENTRE COMPROMETIMENTO E A INTENÇÃO EM DEFENDER A MARCA}

Alguns pesquisadores têm identificado que o comprometimento vem se tornando um fator essencial na formação da intenção comportamental (BROWN et al., 2005). Podendo, aumentar as intenções comportamentais positivas gerando um impacto comportamental efetivo, incluindo a incidência de recompra (ZEITHAML; BERRY; PARASURAMAN, 1996), e ainda, sua possível intenção em defender a marca (ERCIS, et al., 2012). A intenção em defender a marca é a disposição do cliente a dar fortes recomendações e elogios a outros consumidores em nome de um fornecedor de produtos ou serviços (HILL et al., 2006; HARRISON-WALKER, 2001). Portanto, quando os consumidores fornecem recomendações positivas sobre produtos, serviços ou marcas, eles estão agindo como defensores em nome dessa empresa (FULLERTON, 2003).

Um cliente comprometido com a marca normalmente vai agir como um defensor da marca (FULLERTON, 2003). Pesquisadores de marketing descobriram que o compromisso do cliente com a marca ou empresa é associado com as respostas dos clientes, tais como intenção em defender a marca e intenções de recompra (DEAN, 2007).

Suportado por alguns estudos (FULLERTON, 2005; ERCIS, 2012; TURRI; SMITH; KEMP, 2013), comprometimento afetivo e normativo tem uma relação positiva com a intenção em defender a marca, visto que, se o cliente sente um apego emocional afetivo com um prestador de serviço, em seguida, ele estara disposto a agir como um defensor em nome desse prestador de serviço (FULLERTON, 2011). Enquanto, o cliente que se sente em dívida com o parceiro, ou seja, um comprometimento normativo, irá ajudar a empresa, gerando um comportamento favorável, incluindo a recomendação e defesa da empresa e/ou marca (FULLERTON, 2011). Ao passo que, um aumento no comprometimento calculativo levará a um impacto negativo sobre a intenção em defender a marca (FULLERTON, 2011; ERCIS, 2012) pois, quando os clientes se sentem refém da marca, eles tendem a se sentir insatisfeito e, portanto, não defendendo a marca (BALAJI; KUMAR; SADEQUE, 2016). Com base nessa lógica, propõem-se as seguintes hipóteses de pesquisa:

H4: O comprometimento afetivo tem impacto positivo na intenção em defender a marca.

H5: O comprometimento calculativo tem impacto negativo na intenção em defender a marca.

H6: Comprometimento normativo tem impacto positivo na intenção em defender a marca. 


\subsection{RELAÇÃO ENTRE A SATISFAÇÃO DO CLIENTE, INTENÇÃO EM DEFENDER A} MARCA E INTENÇÃO DE RECOMPRA

O comportamento de recompra dos consumidores é considerado um fator importante que pode levar às empresas, à uma maior lucratividade e rentabilidade (FRANK; ENKAWA; SCHVANEVELDT, 2014). Além disso, pode ser considerada um elemento fundamental para o crescimento de mercado para as empresas a partir de sua base atual de clientes (AHMED et al., 2011). Definido por Hellier et al. (2003) como sendo o julgamento ou a predisposição do indivíduo em comprar novamente um determinado produto e/ou serviço de uma mesma empresa, levando em consideração a situação atual e prováveis circunstâncias futuras.

Pesquisas anteriores mostraram que consumidores satisfeitos estão positivamente dispostos a repetir compras com uma mesma empresa (HSU et al., 2014) e mais propensos em permanecer no relacionamento com o fornecedor atual (ANDERSON; SULLIVAN 1993; MOLINARI; ABRATT; DION, 2008). Nesta direção, nota-se que a satisfação do consumidor em relação a uma marca acontece quando o consumidor tem um forte vínculo afetivo com a empresa, podendo adquirir repetidamente o mesmo serviço (LIN; LEKHAWIPAT, 2014). Suportado por alguns estudos (MOLINARI; ABRATT; DION, 2008; CHANG et al., 2013; HSU et al., 2014) verifica-se que a satisfação do cliente apresenta uma associação positiva em relação à intenção de recompra do consumidor. Sendo assim, consumidores que estão satisfeitos com uma marca podem vir a se tornarem consumidores retidos ou leais, adotando um comportamento de compras repetidas (DeLONE; McLEAN, 2003). Assim sendo, é possível apresentar a hipótese 7.

Com relação a intenção em defender a marca, Amoroso e Roman (2015) testaram a relação dessa com a intenção de recompra no contexto de ensino, o estudo demostrou que a intenção em defender a marca é relacionada positivamente com a intenção de recompra e inclusive, a intenção de defesa da marca, pode levar ao aumento das vendas e maiores margens de lucro por parte da empresa. Alguns pesquisadores têm argumentado que a intenção em defender a marca é um indicador relacionado à lealdade do consumidor, podendo levá-lo ao real comportamento de compra repetida, porque o consumidor só defendera um produto, serviço, marca ou empresa quando apresentar sentimentos fortes sobre as mesmas (MAZZAROL et al., 2007; REICHHELD, 2003). Com base nas discussões apresentadas, a seguintes hipóteses são propostas: 
H7: A satisfação do cliente apresenta efeito positivo sobre a intenção de recompra.

H8: A intenção em defender a marca apresenta efeito positivo sobre a intenção de recompra.

\subsection{O EFEITO MODERADOR DO GÊNERO}

Em muitos estudos de marketing, o gênero aparece como uma variável moderadora (SAAD; GILL, 2000; WALSH; EVANSCHITZKY; WUNDERLICH, 2008), podendo afetar as decisões de compras, e até mesmo, as de recompra (HARRIS, 997; MITTAL; KAMAKURA, 2001). No entanto, em pesquisas na aréa do marketing, homens e mulheres devem ser tratados como entidades diferentes (GREWAL; LEVY; LEHMANN, 2003). Inclusive, estudos mostraram que a relação entre a satisfação do cliente e a intenção de recompra é moderada pelo gênero (MITTAL; KAMAKURA, 2001; HOMBURG; GIERING, 2001).

Um estudo realizado por Mittal e Kamakura (2001) na indústria automobilística, nos Estados Unidos, descobriu que a relação entre a satisfação do cliente e o comportamento de repetição de compra é mais forte para os homens do que para as mulheres. Os homens que estão satisfeitos são mais ansiosos para repetir a compra do que as mulheres que estão satisfeitas. Frank, Enkawa e Schvaneveldt (2014) explicaram esta diferença com base na teoria do gênero: "os homens são influenciados para cumprir papéis mais instrumentais, enquanto as mulheres são influenciadas para cumprir papéis mais expressivos". Neste sentido, forma-se a hipótese 9.

Em continuidade, Brannon (1999) acredita que as mulheres são mais provável em se envolver na diciminação do boca a boca do que os homens, e ainda, elas são mais propensas do que os homens em partilhar experiências positivas de um produto e/ou serviço a outras pessoas. O estudo desenvolvido por Cho e Rutherford (2011) em um contexto de estética, suportou a relação entre a intenção do cliente em defender a marca, desiminar a propaganda boca a boca e a intenção de recompra é moderada pelo gênero. Em conclusão, o estudo demosntrou a diferença significativa entre os gêneros, no qual, as mulheres tendem a repetir novamente as compras, ou seja, quando as mulheres estão mais envolvidas na defesa da marca, mais elas são propensas a repetir as compras. Aliás, o consumidor só se envolverá na defesa da marca, quando ele tiver a intenção em repetir a compra (MAZZAROL et al., 2007). Com base nessa lógica, propõe-se a hipótese 10. 
H9: O gênero (masculino e femenino) modera a relação entre a satisfação do cliente e a intenção de recompra.

H10: O gênero (masculino e femenino) modera a relação entre a intenção em defender a marca e a intenção de recompra.

A partir da revisão da literatura, a Figura 1 apresenta o Modelo Teórico proposto:

Figura 1 Modelo Proposto

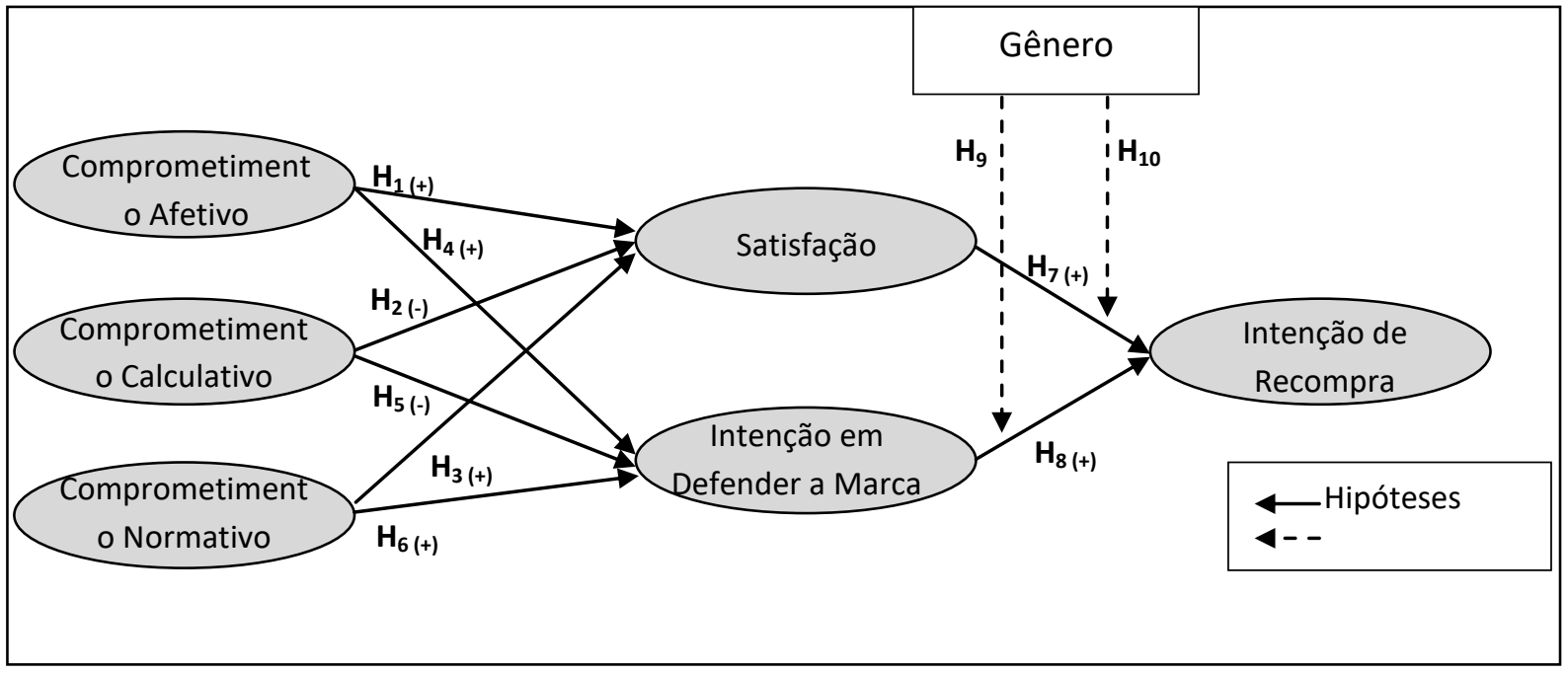

Fonte: Elaborada pelo autor, com base nos autores Shukla, Banerjee e Singh (2016).

\section{MÉTODO DE PESQUISA}

O método de pesquisa utilizado no desenvolvimento da presente pesquisa é de natureza quantitativa, sendo caracterizada como uma pesquisa descritiva, mais notadamente um levantamento (survey) com corte transversal (HAIR Jr. et al., 2010; MALHOTRA; BIRKS; WILLS, 2012).

A amostra foi composta por duzentos e oitenta e cinco (285) alunos de graduação do curso de adminstração de uma Universidade de ensino superior, situada em Caxias do Sul, RS, sendo que 132 (46,3\%) dos participantes da pesquisa são do sexo feminino e $153(53,7 \%)$ do sexo masculino. Salienta-se, portanto, que a seleção da IES e de seus alunos foi por amostragem não-probabilística por conveniência (MALHOTRA; BIRKS; WILLS, 2012). 


\subsection{OPERACIONALIZAÇÃO DOS CONSTRUTOS E ELABORAÇÃO DO} INSTRUMENTO DE COLETA DE DADOS

O instrumento de coleta, foi elaborado com base em escalas validadas, adaptadas ao contexto em estudo, para cada um dos construtos a serem testados, observando-se as contribuições extraídas da revisão bibliográfica. O estudo teve como base o instrumento de pesquisa utilizado pelos autores Shukla, Banerjee e Singh (2016), para o contexto de marcas de luxo, vale resaltar, que foi incluido o construto intenção de recompra, e sua respectiva escala. No qual, o comprometimento afetivo e normativo foi empregada uma escala adaptada de Allen e Meyer (1990) e Jones et al. (2007), enquanto o comprometimento afetivo foi composta por três itens, o calculativo utilizou quatro itens. Para o comprometimento normativo, por sua vez, foi adaptada a escala utilizada por Bansal, Irving e Taylor (2004), composta por cinco itens.

Em relação ao construto satisfação do cliente foi adaptada a escala de Heitmann et al. (2007) e Oliver (1997), composta por 4 itens. Para o construto intenção em defender a marca foi adaptada a escala de Fullerton (2005), composta por três itens. E por fin, para mensurar a intenção de recompra, foi utilizada uma escala composta por quatro itens, adaptadas do estudo de Jones et al. (2007), Zeithaml et al.(1996) e Mittal, Ross e Baldasar (1988), vide Figura 1. É importante salientar que todas as escalas passaram pela tradução reversa (backtranslation). Os questionários foram estruturados com perguntas fechadas, no qual utilizou-se uma escala do tipo Likert de sete pontos, tendo em seus extremos "1. Discordo Totalmente" à "7. Concordo Totalmente", com um pré-teste (27 questionários). Quanto ao método de coleta dos dados foi utilizada a abordagem pessoal mediante a técnica de autopreenchimento (MALHOTRA, BIRKS, WILLS, 2012).

O instrumento de coleta de dados, depois de estruturado, passou por uma validação de face e de conteúdo (HAIR, Jr. et al., 2009) em que o instrumento foi submetido para três experts da área Após este refinamento o instrumento foi pré-testado junto a 26 alunos, cujas características eram iguais ao público alvo desta pesquisa (alunos da IES investigada), sendo que não houve necessidades de ajustes que necessitasse um ajuste da escala. 
Figura 2 Escalas utilizadas para mensuração dos construtos

\begin{tabular}{|c|c|c|}
\hline Construto & Variável & $\begin{array}{l}\text { Escala de } \\
\text { referência }\end{array}$ \\
\hline $\begin{array}{c}\text { Comprometimento } \\
\text { Afetivo }\end{array}$ & $\begin{array}{l}\text { Esta universidade tem um grande significado pessoal para } \\
\text { min. } \\
\text { Eu sinto um senso de pertencimento a esta universidade. } \\
\text { Eu sou aluno desta universidade porque eu realmente } \\
\text { gosto. }\end{array}$ & $\begin{array}{c}\text { Allen e } \\
\text { Meyer (1990) } \\
\text { Jones et al. } \\
(2007)\end{array}$ \\
\hline $\begin{array}{l}\text { Comprometimento } \\
\text { Calculativo }\end{array}$ & $\begin{array}{l}\text { Seria muito difícil deixar esta universidade neste } \\
\text { momento, mesmo que eu quisesse. } \\
\text { Muito na minha vida seria rompido se eu decidisse deixar } \\
\text { neste momento esta universidade. } \\
\text { Estar com esta universidade, neste momento, é uma } \\
\text { questão de necessidade tanto quanto desejo. } \\
\text { Sinto-me de alguma forma obrigado a estudar nesta } \\
\text { universidade. }\end{array}$ & $\begin{array}{c}\text { Allen e } \\
\text { Meyer (1990) } \\
\text { Jones et al. } \\
(2007)\end{array}$ \\
\hline $\begin{array}{c}\text { Comprometimento } \\
\text { Normativo }\end{array}$ & $\begin{array}{l}\text { Se tivesse oportunidade de estudar em outro lugar, eu não } \\
\text { sentiria que seria certo deixar esta universidade. } \\
\text { Mesmo que fosse vantagem pra mim, eu não sinto que é } \\
\text { certo deixar esta universidade. } \\
\text { Eu não deixaria esta universidade agora, por que eu tenho } \\
\text { um grande senso de obrigação com ela. } \\
\text { A universidade merece minha lealdade. } \\
\text { Eu me sentiria culpado se deixasse esta universidade } \\
\text { agora. }\end{array}$ & $\begin{array}{c}\text { Bansal Irving } \\
\text { e Taylor } \\
\text { (2004) }\end{array}$ \\
\hline $\begin{array}{l}\text { Satisfação do } \\
\text { Cliente }\end{array}$ & $\begin{array}{l}\text { Eu realmente gosto de estudar nesta universidade. } \\
\text { Esta universidade me proporciona exatamente o que eu } \\
\text { preciso. } \\
\text { Estudar nesta universidade tem sido uma boa experiência. } \\
\text { Estou satisfeito com minha decisão de estar estudando } \\
\text { nesta universidade. }\end{array}$ & $\begin{array}{l}\text { Heitmann et } \\
\text { al. (2007) e } \\
\text { Oliver (1997) }\end{array}$ \\
\hline $\begin{array}{c}\text { Intenção em } \\
\text { Defender a Marca }\end{array}$ & $\begin{array}{l}\text { Eu buscarei dizer coisas positivas sobre a universidade } \\
\text { para outras pessoas. } \\
\text { Eu recomendarei a instituição para pessoas que buscam } \\
\text { meu conselho. } \\
\text { Eu encorajarei amigos e conhecidos a estudar nesta } \\
\text { universidade. }\end{array}$ & $\begin{array}{l}\text { Fullerton } \\
\text { (2005) }\end{array}$ \\
\hline $\begin{array}{l}\text { Intenção de } \\
\text { Recompra }\end{array}$ & $\begin{array}{l}\text { Planejo continuar estudando nesta universidade no futuro. } \\
\text { Considerarei esta universidade como minha primeira } \\
\text { opção para a matrícula em cursos de extensão e/ou } \\
\text { especialização. } \\
\text { No futuro, se eu realizar um curso de pós-graduação, } \\
\text { escolherei esta universidade em detrimento da } \\
\text { concorrência. } \\
\text { Eu pretendo adquirir outros serviços desta universidade, no } \\
\text { futuro. }\end{array}$ & $\begin{array}{l}\text { Jones et al. } \\
\text { (2007), } \\
\text { Zeithaml et } \\
\text { al. (1996) e } \\
\text { Mittal, Ross e } \\
\text { Baldasar } \\
\quad(1988)\end{array}$ \\
\hline
\end{tabular}




\subsection{PREPARACAO DOS DADOS}

Em se tratando dos missings (dados perdidos), foi adotado o procedimento conhecido por abordagem de caso completo (listwise deletion) (ENDERS, 2010) nesta abordagem formam excluídos 5 casos. Quanto os outliers, a partir das observações atípicas univariadas ( $Z$ scores) e outliers multivariados (teste da distância de Mahalanobis $\mathrm{D}^{2}$ ) não foi identificado nenhum caso de outliers. Permanecendo o tamanho da amostra final de 280 casos. Também foram realizados os testes de normalidade, linearidade e multicolinearidade, onde os valores se encontram dentro do permitido (HAIR Jr. et al., 2009).

\subsection{ANÁLISE DO MODELO DE MENSURAÇÃO DO MODELO ESTRUTURAL}

Para o teste do modelo, foi realizada a Análise Fatorial Confirmatória, utilizando-se o software SPSS Statistics 20, verificou-se que a estimativa (estimate) foram consideradas satisfatórias para todas as variáveis, uma vez que valores de acima de 0,50 são considerados muito bons (HAIR Jr. et al., 2010). A avaliação da validade convergente foi feita com base na carga fatorial de cada item por construto, no cálculo da confiabilidade composta, ficando com os valores 0,85 (Comprometimento Calculativo) à 0,95 (Intenção em Defender a Marca) dentro dos valores recomendado pela literatura 0,70 . Quanto a variância extraída, os valores foram satisfatórios ficando entre 0,52 (Comprometimento Normativo) à 0,80 (Intenção em Defender a Marca), sendo esses superiores ao mínimo recomendado pelos autores (maior ou igual a 0,50) (HAIR Jr. et al., 2010).

Além disso, foi realizado o cálculo do Alpha de Cronbach para avaliar a confiabilidade das medidas e a consistência interna dos dados, que deve apresentar índices aceitáveis, sendo iguais ou superiores a 0,70 (CHURCHILL Jr., 2001; MALHOTRA; BIRKS; WILLS, 2012), deste modo, o Alpha de Cronbach para os construtos testados apresentou valores entre 0,832 (Comprometimento Afetivo) e 0,922 (Intenção em Defende a Marca). A validade discriminante, por sua vez, seguiu o método de Fornell e Larcker (1981). Para aplicá-lo, uma AFC por máxima verossimilhança foi conduzida para o modelo proposto e seus construtos (Tabela 1).

A partir da MEE, utilizando o método de estimação de máxima verossimilhança, foi possível identificar a qualidade dos ajustes, conforme mostram os resultados da Tabela 2. Diante disso, conforme os valores de referência propostos pela literatura (HAIR Jr. et al., 2009) os resultados são satisfatórios para os índices NFI $(0,900)$, TLI $(0,934)$, CFI $(0,944)$ e RMSEA $(0,630)$. Entretanto, os índices AGFI $(0,844)$, e GFI $(0,879)$ estão abaixo do ideal de 
0,90. Contudo, vale ressaltar que, para modelos complexos, esses índices podem ser aceitos como valores marginais quando acima de 0,80 (HAIR Jr. et al., 2009).

Tabela 1 Validade discriminante

\begin{tabular}{lcccccc}
\hline \multicolumn{1}{c}{ Construtos } & $\mathbf{1}$ & $\mathbf{2}$ & $\mathbf{3}$ & $\mathbf{4}$ & $\mathbf{5}$ & $\mathbf{6}$ \\
\hline 1. Comprometimento Afetivo & $\mathbf{0 , 6 2 6}$ & & & & & \\
2. Comprometimento Calculativo & 0,479 & $\mathbf{0 , 5 4 7}$ & & & & \\
3. Comprometimento Normativo & 0,497 & 0,484 & $\mathbf{0 , 5 2 7}$ & & & \\
4. Satisfação do Cliente & 0,717 & 0,377 & 0,479 & $\mathbf{0 , 7 1 7}$ & & \\
5. Intenção em Defender a Marca & 0,697 & 0,400 & 0,434 & 0,818 & $\mathbf{0 , 8 0 6}$ & \\
5. Intenção de Recompra & 0,658 & 0,407 & 0,439 & 0,723 & 0,738 & $\mathbf{0 , 6 5 8}$ \\
\hline
\end{tabular}

Tabela 2 Índices de ajuste

\begin{tabular}{cc}
\hline Medidas de Ajustes & Valores \\
\hline GFI & 0,879 \\
AGFI & 0,844 \\
RMSEA & 0,630 \\
TLI & 0,934 \\
NFI & 0,900 \\
CFI & 0,944 \\
\hline
\end{tabular}

Em seguida foi realizado o teste das hipóteses. A Tabela 3 apresenta os resultados consolidados dos testes realizados.

Os resultados apresentados na Tabela 3, revelam que das oito hipóteses proposta pelo estudo, duas não foram suportadas. No entanto, podemos identificar que a primeira hipótese proposta $\mathrm{H} 1$, a qual propõe que o comprometimento afetivo (COMP_AFE) esta positivamente associado a satisfação do cliente (SAT), foi sustentada pelos resultados. Para a presente relação, o coeficiente padronizado encontrado foi de 0,914 e t-value $=9,426$, sendo que esta relação também foi evidenciada em estudos anteriores (MATHIEU, 1991; SHUKLA; BANERJEE; SINGH. 2016). Levando em consideração a marca, a comprovação da hipótese H1 evidencia que as IES devem concentrar seus esforços para desenvolver maior satisfação do cliente (aluno), através do comprometimento afetivo, criando uma ligação emocional entre a marca e o aluno. 
Tabela 3 Testes de hipótese do Modelo Teórico

\begin{tabular}{|c|c|c|c|c|c|c|c|}
\hline Hi & $\begin{array}{l}\text { Caminhos } \\
\text { Estruturais }\end{array}$ & $\begin{array}{c}\text { Coeficientes } \\
\text { Não } \\
\text { Padronizados } \\
\text { (b) }\end{array}$ & Erros & $\begin{array}{c}\text { Coeficientes } \\
\text { Padronizados } \\
\text { (ß) }\end{array}$ & $\begin{array}{c}t- \\
\text { values }\end{array}$ & $\mathbf{P}$ & Resultados \\
\hline H1 & $\begin{array}{c}\text { COMP_AFE } \rightarrow \\
\text { SAT }\end{array}$ & 0,011 & 0,117 & 0,914 & 9,426 & $\mathrm{p}<0,001$ & Suportada \\
\hline $\mathrm{H} 2$ & $\underset{\text { SAT }}{\text { COMP_CAL }} \rightarrow$ & 0,017 & 0,049 & 0,020 & 0,346 & $\mathrm{p}=0,729$ & $\begin{array}{c}\text { Não } \\
\text { Suportada }\end{array}$ \\
\hline $\mathrm{H} 3$ & $\underset{\text { SAT }}{\text { COMP_NORM }} \rightarrow$ & 0,283 & 0,082 & 0,218 & 3,470 & $\mathrm{p}<0,001$ & Suportada \\
\hline $\mathrm{H} 4$ & $\begin{array}{l}\text { COMP_AFE } \rightarrow \\
\text { INT_DEFESA }\end{array}$ & 0,957 & 0,102 & 0,848 & 9,364 & $\mathrm{P}<0,001$ & Suportada \\
\hline H5 & $\begin{array}{l}\text { COMP_CAL } \rightarrow \\
\text { INT_DEFESA }\end{array}$ & 0,043 & 0,046 & 0,056 & 0,935 & $\mathrm{p}=0,350$ & $\begin{array}{c}\text { Não } \\
\text { Suportada }\end{array}$ \\
\hline H6 & $\begin{array}{l}\text { COMP_NORM } \rightarrow \\
\text { INT_DEFESA }\end{array}$ & 0,164 & 0,073 & 0,134 & 2,231 & $\mathrm{p}=0,026$ & Suportada \\
\hline $\mathrm{H} 7$ & $\mathrm{SAT} \rightarrow \mathrm{INT}$ RECP & 0,486 & 0,112 & 0,467 & 4,319 & $\mathrm{P}<0,001$ & Suportada \\
\hline $\mathrm{H} 8$ & $\begin{array}{c}\text { INT_DEFESA } \rightarrow \\
\text { INT_RECP }\end{array}$ & 0,382 & 0,116 & 0,344 & 3,289 & $\mathrm{P}<0,001$ & Suportada \\
\hline
\end{tabular}

Foi observado que o comprometimento calculativo (COMP_CAL) não impacta positivamente na satisfação do cliente $(\mathrm{SAT})$, (coeficiente padronizado $=0,020$ e $t$-value $=$ 0,346), confirmando a H2. Observa-se que no contexto analisado, o comprometimento calculativo não seja susceptível em desenvolver uma forte ligação com a satisfação do cliente (aluno), pelo fato do comprometimento calculativo aproximar o aluno a IES, pela percepção dos custos de deixar o relacionamento existente (GUSTAFSSON; JOHNSON; ROOS, 2005; SHUKLA; BANERJEE; SINGH, 2016). A hipótese H3 que indica que a satisfação do cliente (SAT) é impactada positivamente pelo comprometimento normativo (COMP_NORM), foi corroborada, apresentando coeficiente padronizado $=0,218$ e $t$-value $=3,470$. $\mathrm{O}$ fato de o aluno demosntrar um comprometimento normativo com a IES de vínculo, motivado pela obrigação moral em apoiar a IES, gera sua satisfação, uma vez, que existe a reciprocidade entre o aluno e a IES.

A hipótese H4, que propõe uma relação positiva entre o comprometimento afetivo (COMP_AFE), e a intenção de defesa da marca (INT_DEFESA), foi confirmada. Essa relação apresentantou coeficiente padronizado $=0,848$ e $t$-value $=9,364$. No que diz respeito à esta relação, registra-se que o comprometimento afetivo pode servir como um elemento fundamental na intenção do aluno defender a marca da IES. Neste sentido, nota-se que os clientes (alunos) que possuem um forte vínculo afetivo com uma IES podem vir, inclusive, a adotar um comportamento de defesa da marca (ERCIS, 2012). 
Em relação, ao comprometimento calculativo (COMP_CAL) e a intenção em defender a marca (INT_DEFESA) não foi suportada (coeficiente padronizado $=0,056$ e $t$-value $=0,935$ ), confirmando a hipotese H5. Quando o aluno se sente obrigado em permanecer na IES de vínculo, levando em conta a percepção de custo, ele tende a se sentir insatisfeito e, portanto, não defendendo a marca (BALAJI; KUMAR; SADEQUE, 2016). Em continuidade, verificase que a hipótese H6, a qual, testou a relação entre o comprometimento normativo (COMP_NORM) e a intenção em defender a marca (INT_DEFESA), foi suportada, apresentado coeficiente padronizado $=0,134$ e $t$-value $=2,231$. É relatado que, quando o aluno se sente em dívida com o a IES, ele agira a favor da IES, defendendo a marca da instituição.

Quando observada a relação entre satisfação do cliente (SAT) e a intenção de recompra (INT_RECP), a hipótese H7, que trata dessa relação foi sustentada (coeficiente padronizado $=-$ 0,467 e t-value $=4,319)$. Esse suporte é encontrado em estudos anteriores (MOLINARI; ABRATT; DION, 2008; CHANG et al., 2013; HSU et al., 2014), sendo que, no contexto analisado, observa-se que o aumento da satisfação do cliente (aluno) com a IES, incide na intenção do aluno recomprar novos serviços, tais como, cursos de extensão, especialização e pós-graduação. Ainda observando a Intenção de recompra (INT_RECP), quando relacionada com a intenção em defender a marca (INT_DEFESA), verifica-se uma realação positiva entre os construtos (coeficiente padronizado $=0,344$ e t-value $=3,289$ ), sustentando a hipótese H8. Esta relação pode ser vista pelo fato, que quando o aluno está disposto a defender a marca da IES, ele esteja disposto a continuar estudando na Universidade.

Considerando o coeficiente de determinação $\left(\mathrm{R}^{2}\right)$, há um poder explicativo de $59,6 \%$ da intenção de recompra. Isso indica que variações na intenção de recompra estão fortemente associadas aos quatro construtos antecedentes. Observou-se, uma forte explicação da satisfação do cliente ( $\mathrm{R}^{2}$ de $\left.88,3 \%\right)$ e a intenção em defender a marca $\left(\mathrm{R}^{2}\right.$ de $\left.74 \%\right)$ a partir do seus antecedentes (compometimento afetivo, calculativo e normativo).

\subsection{ANÁLISE DO EFEITO MODERADOR}

A análise a seguir permitiu avaliar o efeito moderador que o gênero pode impactar na relação "satisfação do cliente e intenção de recompra" e "intenção em defender a marca e intenção de recompra". Com este objetivo, aplicou-se a análise de multigrupos, dividindo a amostra em dois grupos (BYRNE, 2009), sendo um, composto por $151(53,9 \%)$ alunos do 
sexo masculino e outro, formado por $129(46,1 \%)$ alunos do sexo feminino. Os resultados destes cruzamentos são apresentados na Tabela 4.

Tabela 4 Teste de moderação do gênero

\begin{tabular}{ccccccc}
\hline \multirow{2}{*}{ Hi } & Caminhos Estruturais & \multicolumn{2}{c}{ Gênero Masculino } & \multicolumn{2}{c}{ Gênero Feminino } & \multirow{2}{*}{ ZScores } \\
& & Estimate & Sig. & Estimate & Sig. & \\
\hline H9 & SAT $\rightarrow$ INT_RECP & 0,548 & 0,000 & 0,353 & 0,010 & 0,493 \\
H10 & INT_DEFESA $\rightarrow$ INT_RECP & 0,411 & 0,008 & 0,511 & 0,000 & $-0,957$ \\
\hline
\end{tabular}

Obs.: ${ }^{*} \mathrm{p}<0,05$.

No teste da hipótese H9, verificou-se que o gênero não teve uma diferença significativa ao nível de $\mathrm{p}<0,05$ na moderação. Neste caso, os diferentes gêneros não moderaram a relação entre a satisfação do cliente e a intenção de recompra. Da mesma forma, quando observado se a relação entre a intenção em defender a marca e intenção de recompra é moderado pelo gênero, a hipótese H10, não foi suportda, ou seja, o gênero demontra não estar moderadorando esta relação.

\section{CONSIDERAÇÕES FINAIS, LIMITAÇÕES E SUGESTÕES DE FUTURAS PESQUISAS}

Estudo que contribui teoricamente, ao acrescentar às limitações e futuras pesquisas, sugeridas por Shukla, Banerjee e Singh (2016), a utilização do construto intenção de recompra e a replicação do modelo à marcas não de luxo. Neste estudo foi constatado que as hipóteses não-significativas, também foram confirmadas no estudo de Shukla, Banerjee e Singh (2016). Estes autores (2016) justificaram a rejeição da relação entre comprometimento calculativo e a satisfação do cliente, e o comprometimento calculativo e a intenção de defender a marca, como uma sensação de refém à marca, que resulta na insatisfação, o que contribui para não defender a marca e não recomprar um novo serviço desta marca. Portanto, é possível constatar que este estudo no contexto de IES também existe falta de relação, não-significância entre os construtos em estudo.

Outra contribuição diz respeito à proposição do efeito moderador do gênero nas relações entre os construtos consequentes do comprometimento e a intenção de recompra. As moderações não apresentaram diferenças significativas. Esse resultado não está alinhado com 
o resultado verificado na pesquisa de Mittal e Kamakura (2001), que identificou diferenças entre respondentes de gêneros distintos em relação à satisfação do cliente e a intenção de recompra. O resultado desse estudo também contraria os achados de Cho e Rutherford (2011) que verificaram diferenças estatísticas significativas entre respondentes de gêneros distintos em relação à intenção em defender a marca e intenção de recompra. O presente estudo contribui, portanto, para realçar que neste contexto, ambos os gêneros, não apresentam diferenças significativas nas suas intenções comportamentais, estimulando novas pesquisas sobre o assunto.

Como contribuições gerenciais, com base nos resultados encontrados é possível constatar que o comprometimento afetivo é o construto que mais impacta na intenção de defender uma marca e a satisfação do cliente, responsáveis por representar a explicação da satisfação do cliente e intenção de defender a marca, neste modelo. Portanto, através destes resultados é importante que as IES's tenham a compreensão de gerar um comprometimento afetivo do usuário do serviço com a instituição, contribuindo para a realização de recompra de serviços de uma IES. Ou seja, os gestores das Universidades devem estar atentos para estas questões, para fomentar a intenção de recompra de novos serviços junto aos alunos. Adicionalmente, o estudo tem o intuito de alertar os executivos sobre o fato de que não existe diferença do gênero, nas intenções comportamentais dos alunos.

Referente as limitações de estudo, como se observa na Tabela 1, os resultados indicaram que a variância extraída do construto Comprometimento Afetivo $(0,626)$ apresentou-se inferior à variância compartilhada com o construto Satisfação do cliente $(0,717)$, Intenção em Defender a Marca $(0,697)$ e Intenção de Recompra $(0,658)$. A variância extraída do construto Intenção em Defender a Marca $(0,806)$ também apresentou à variância compartilhada inferior ao construto Intenção de Recompra $(0,738)$, evidenciando uma possível redundância entre os construtos pelos mesmos estarem fortemente correlacionados.

De qualquer modo, optou-se por manter o construto Comprometimento Afetivo e Satisfação do cliente pela sustentação teórica do modelo proposto. Sugestões de futuros estudos, recomenda-se o melhoramento/refinamento das escalas, pois algumas variáveis apresentaram baixa validade discriminante. Além do mais, como estudos futuros recomendase a comparação entre cursos da mesma IES ou um comparativo entre instituições de ensino superior. 


\section{REFERÊNCIAS}

AMPARO SOBRINHO, J.B. Contrato psicológicos de professores de ensino superior de instituições públicas e particulares. Dissertação de mestrado em psicologia. UFBA - PPGP, Salvador, 2011.

BAL, P.M., \& SMIT, P. The older the better!: Age-related differences in emotion regulation after psychological contract breach. Career Development International. 17(1), 6-24, 2012.

BAL, P.M., CHIABURU, D.S., \& DIAZ, I. Does psychological contract breach decrease proactive behaviors? The moderating effect of emotion regulation. Group \& Organization Management, 36(6), 722-758, 2011.

BAO, Y., OLSO, B., PARAYITAM, S., \& ZHAO, S.The effects of psychological contract violation on Chinese executives. The International Journal of Human Resource Management, Vol 22(16), 2011.

BASTOS, A.V.B., MAIA, L.G., RODRIGUES, A.C.A., MACAMBIRA, M.O., \& BORGESANDRADE, J.E.Vínculos dos Indivíduos com a Organização: Análise da Produção Científica Brasileira 2000-2010. Psicologia: Teoria e Pesquisa, Abr-Jun, Vol. 30 n. 2, pp. 153-162, 2014.

BORDIA, P., RESTUBOG, S.L.D., \& TANG, R.L.When employees strike back: investigating mediating mechanisms between psychological contract breach and workplace deviance. Journal of Applied Psychology, Vol 93(5), Sep, p.1104-1117, 2008.

BYRNE, B.M. Structural equation modelong with AMOS: Basic concepts, applications, and programming. New York, NY: Routledge, Taylor \& Francis, 2010.

CARVALHO, C.H.A. Política para o ensino superior no Brasil (1995-2008). Ruptura e continuidade nas relações entre público e privado. In: J. R. S., SILVA, J. F. OLIVEIRA \& D. MANCEBO. (Orgs.) Reforma universitária: dimensões e perspectivas. Campinas: Alínea, p. 125-139, 2006.

CAVANAUGH, M., \& NOE, R. Antecedents and consequences of relational components of the new psychological contract. Journal of Organizational Behavior, Vol. 20 (3), 323$340,1999$.

CHIN, P.L., \& HUNG, M.L. Psychological contract breach and turnover intention: the moderating roles of adversity quotient and gender. Social Behavior and Personality. Vol. 41, No.5 pp843-860, 2013.

CHIUZI, R.M., \& MALVEZZI, S. Mais de 50 anos de pesquisa sobre contratos psicológicos de trabalho: sistematização teórica e sugestão de agenda futura de pesquisa. Revista de Carreiras e Pessoas São Paulo. Volume IV - Número 03 - Set/Out/Nov/Dez 2014, pp. $282-294,2014$. 
CHIUZI, R.M. As dinâmicas de formação e continuidade dos contratos psicológicos de trabalho. (Tese de doutorado, Instituto de Psicologia, USP), 2014.

CHU, H., \& KUO, T. Exploring Faculty Psychological Contract through leadership style and institutional climate in a higher education setting. International Journal of Business and Social Science. Vol. 3, No.4 pp159-164, 2012.

CONWAY, N., \& BRINER, R.B. A daily diary study of affective responses to psychological contract breach and exceeded promises. Journal of Organizational Behavior, Vol. 23, 287-302, 2002.

COOPER, C.L. A natureza mutante do trabalho: o novo contrato psicológico e os estressores associados. In A.M. Rossi, P.L. Perrewé, \& S. L. Sauter (Orgs.) Stress e Qualidade de Vida no Trabalho (pp.3-8). São Paulo: Atlas, 2008.

CORRAR, L.J., PAULO, E., \& FILHO, J.M.D. (ORGS.). Análise Multivariada. São Paulo: Atlas, 2007.

DABOS, G. E., \& ROUSSEAU, D. M. Mutuality and reciprocity in the psychological contracts of employees and employers, Journal of Applied Psychology, 89 (1), 52-72, 2004.

FARNSWORTH, E.A. Contracts. Boston: Little Brown, 1982.

FRANCO, A. P. Ensino Superior no Brasil: cenário, avanços e contradições. Jornal de Políticas educacionais, 4, 53-63, 2008.

GAMMIE, R. P. Psychological Contracts in a Business School Context. (Tese de doutorado) University Of Starling, 2006.

GOULDNER, A. W. The norm of reciprocity: A preliminary statement. American Sociological Review, 25, 161-178, 1960.

GUEST, D., \& CONWAY, N. Communicating the psychological contract: An employer perspective. Human Resource Management Journal,12, 22-38, 2002.

HAIR, J.F., BLACK, W.C., BABIN, B.J., ANDERSON, R.E., \& TATHAM, R.L. Análise Multivariada de dados. Porto Alegre: Bookman, 2010.

HAO ZHAO, WAYNE, S.J., GLIBKOWSKI, B.C., \& BRAVO, J. The impact of psychological contract breach on work-related outcomes: a meta-analysis. Personnel Psychology, 60, 640-680, 2007.

HERRIOT, P., MANNING, W. E. G., \& KIDD, J. M. The content of the psychological contract. British Journal of Management, 8, 151-162, 1997.

INEP - Instituto Nacional de Estudos e Pesquisas Educacionais Anísio Teixeira. Censo da educação superior, Brasilia, 2014. Recuperado em 23/04/2015 em http://portal.inep.gov.br/web/censo-da-educacao-superior 
JAMIL, A.; RAJA, U., \& DARR, W. Psychological Contract Types as Moderator in the Breach-Violation and Violation-Burnout Relationships. The Journal of Psychology, 147(5), 491-515, 2013.

JANSSENS, M.; SELS, L., \& VAN DEN BRANDE, I. Multiple types of psychological contracts - a six-cluster solution. Human Relations, 56 (11). 1349-1378, 2003.

KICKUL, J. When organizations break their promises: Employee reactions to unfair processes and treatment. Journal of Business Ethics, 29, 289-307, 2001.

KLINE, R.B. Principles and practice of structural equation modelling. New York, NY: Guilford Press, 2011.

KRIVOKAPIC-SKOKO, B., \& O’NEILL, G. University Academics’ Psychological Contracts in Australia: a Mixed Method Research Approach. The Electronic Journal of Business Research Methods, 6 (1) 61-72, 2008.

LAPALME, M.È.; SIMARD, G., \& TREMBLAY, M. The influence of psychological contract breach on temporary workers' commitment and behaviors: A multiple agency perspective. Journal of Business and Psychology, Vol 26(3), 311-324, 2011.

LAUS, S., \& MOROSINI, M.C. Internacionalización de la educación superior en Brasil, Colombia: Banco Mundial en coedición con Mayol Ediciones, 2005.

LITTLE, T. D., CUNNINGHAM, W. A., SHAHAR, G., \& WIDAMAN, K. F. To parcel or not to parcel: Exploring the question, weighing the merits. Structural Equation Modeling, 9, 151-173, 2002.

MARÔCO, J. Análise de equações estruturais. Pêro Pinheiro, Portugal: Report Number, 2010.

MARTINS, C.B. A reforma universitária de 1968 e a abertura para o ensino superior privado no Brasil. Educ. Soc., Campinas, vol. 30, n.106, p.15-35, jan./abr, 2009.

MENEGON, L.F., \& CASADO, T. O contrato psicológico como ferramenta para a gestão de pessoas. Revista de Administração., São Paulo, 41 (2), 125-135, 2006.

NUNES, E.O. Educação superior no Brasil: estudos, debates e controvérsias. Rio de Janeiro: Garamond, 2012.

PASQUALI, L. Psicometria: Teoria dos testes na Psicologia e na Educação. Rio de Janeiro: Vozes, 2003.

RIOS, M. C., \& GONDIM, S. M. G. Contrato psicológico de trabalho e a produção acadêmica no Brasil. Revista Psicologia: Organizações e Trabalho, 10(1), 23-363 2010. 
RIOS, M.C., \& GONDIM, S.M.G. Contratos Psicológicos e Terceirização: Um estudo das relações entre vínculos e as práticas de gestão de pessoas, Organizações \& Sociedade vol.17 no.55, 2010.

ROBINSON, S. L., \& MORRISON, E. W. The development of psychological contract breach and violation: a longitudinal study. Journal of Organizational Behavior, 21, 525$546,2000$.

ROUSSEAU, D.M. (1995). Psychological contracts in organizations - understanding written and unwritten agreements Thousand Oaks, CA: Sage.

SEVERINO, A.J. O ensino superior brasileiro: novas configurações e velhos desafios. Educar, Curitiba, n. 31, p. 73-89, Editora UFPR, 2008.

SHEN, J. University academics' psychological contracts and their fulfillment. Journal of Management Development, 29 (6), 575-591, 2010.

STAlliVIERI, L. O Sistema de Ensino Superior do Brasil: Características, Tendências e Perspectivas. Educación superior en América Latina y el Caribe: Sus estudiantes hoy. México, 2007.

TIPPLES, R., KRIVOKAPIC-SKOKO, B., \& O’NEILL, G. University Academics' Psychological Contracts in Australia and New Zealand. New Zealand Journal of Employment Relations. 32(2): 32 - 52, 2007.

WADE-BENZONI, K., ROUSSEAU, D., \& LI, M. Managing relationships across generations of academics. International Journal of Conflict Management. 17(1): 4-33, 2006

WELLIN, M. Managing the Psychological Contract: Using the personal deal to increase business performance. Cornwall: MPG Books, 2007. 